\title{
A way toward ventilator-associated lower respiratory tract infection research: reply
}

\author{
Shannon M. Fernando ${ }^{1,2^{*}}$, Michael Klompas ${ }^{3,4}$ and Bram Rochwerg ${ }^{5,6}$
}

๑) 2020 Springer-Verlag GmbH Germany, part of Springer Nature

\section{Dear Editor,}

We thank Martin-Loeches et al. [1] for their thoughtful comments on our systematic review and meta-analysis evaluating the accuracy of clinical signs and symptoms for the diagnosis of ventilator-associated pneumonia (VAP) in critically ill adults [2]. We agree with many of the points expressed by the authors, and indeed noted many of the same concerns within our manuscript, leading us to rate the quality of evidence supporting the diagnostic accuracy of each sign or symptom as "low" or "very low."

We agree that histopathology is an imperfect reference standard for diagnosis of VAP, due to both the potential for sampling error as well as interobserver variability between pathologists [3]. That being said, what other reference standard is there to choose that is associated with less uncertainty? Clinical consensus, quantitative cultures, radiological readings, and diagnostic criteria are also all prone to similar if not greater variation due to differences in measurement approaches and interpretation [3-5]. We believe histology is the best reference standard available among an admittedly very imperfect set of options.

We also agree with Martin-Loeches and colleagues that in actual practice, clinicians are more likely to use a combination of clinical signs rather than any single finding in isolation. We were unable to perform a meta-analysis on combinations of findings because no two studies evaluated the accuracy of the same combinations of findings in the same fashion. We therefore did the next best thing which was to report the results of each of the individual

\footnotetext{
*Correspondence: sfernando@qmed.ca

${ }^{2}$ Department of Emergency Medicine, University of Ottawa, Ottawa, ON, Canada
}

Full author information is available at the end of the article studies evaluating the accuracy of combinations of findings (summarized in Table 3 of our paper).

Martin-Loeches and colleagues rightly point out that ventilator-associated event (VAE) criteria were not designed for clinical diagnosis, but rather for surveillance alone [6]. We agree. The reason we mentioned VAE was not to suggest that it might serve as a substitute for clinical diagnostic criteria for VAP, but rather to indicate how the limitations of clinical diagnostic criteria led Centers for Disease Control and Prevention to seek a different strategy for measuring quality of care for ventilated patients.

Finally, Martin-Loeches and colleagues note the interconnections between ventilator-associated tracheobronchitis (VAT) and VAP. This was beyond the scope of our review, as we focused on VAP alone rather than VAT, although we suspect that many of the same limitations we documented for the common clinical criteria used to diagnose VAP may also apply to using these same signs to diagnose VAT.

All told, we continue to recommend caution when trying to diagnose VAP given the limited association between common diagnostic criteria, both alone and in combination, with histologically confirmed pneumonia. In practice, clinicians have little choice but to use these clinical signs to manage patients, but we continue to recommend tempering them with diagnostic humility, thoughtful use of cross-sectional imaging, calibrating treatment decisions to patients' severity of illness, and continually reassessing the appropriateness of a diagnosis for or against VAP in light of a patient's clinical trajectory and response to treatment.

\footnotetext{
Author details

${ }^{1}$ Division of Critical Care, Department of Medicine, University of Ottawa, Ottawa, ON, Canada. ${ }^{2}$ Department of Emergency Medicine, University of Ottawa, Ottawa, ON, Canada. ${ }^{3}$ Department of Population Medicine,
} 
Harvard Medical School, Boston, MA, USA. ${ }^{4}$ Division of Infectious Diseases, Brigham and Women's Hospital, Boston, MA, USA. ${ }^{5}$ Division of Critical Care, Department of Medicine, McMaster University, Hamilton, ON, Canada.

${ }^{6}$ Department of Health Research Methods, Evidence, and Impact, McMaster University, Hamilton, ON, Canada.

\section{Compliance with ethical standards}

\section{Conflicts of interest}

None of the authors report any relevant conflict of interest.

\section{Publisher's Note}

Springer Nature remains neutral with regard to jurisdictional claims in published maps and institutional affiliations.

\section{Accepted: 23 May 2020}

Published online: 4 June 2020

\section{References}

1. Martin-Loeches I, Povoa P, Nseir S (2020) A Way Towards Ventilator-Associated Lower Respiratory Tract Infection Research. Intensive Care Med. https://doi.org/10.1007/s00134-020-06101-7
2. Fernando SM, Tran A, Cheng W, Klompas M, Kyeremanteng K, Mehta S, English SW, Muscedere J, Cook DJ, Torres A, Ranzani OT, Fox-Robichaud AE, Alhazzani W, Munshi L, Guyatt GH, Rochwerg B (2020) Diagnosis of ventilator-associated pneumonia in critically ill adult patients-a systematic review and meta-analysis. Intensive Care Med. https://doi. org/10.1007/s00134-020-06036-z

3. Klompas M (2007) Does this patient have ventilator-associated pneumonia? JAMA 297:1583-1593

4. Browne E, Hellyer TP, Baudouin SV, Conway Morris A, Linnett V, McAuley DF, Perkins GD, Simpson AJ (2014) A national survey of the diagnosis and management of suspected ventilator-associated pneumonia. BMJ Open Respir Res 1:e000066

5. Ego A, Preiser JC, Vincent JL (2015) Impact of diagnostic criteria on the incidence of ventilator-associated pneumonia. Chest 147:347-355

6. Magill SS, Klompas M, Balk R, Burns SM, Deutschman CS, Diekema D, Fridkin S, Greene L, Guh A, Gutterman D, Hammer B, Henderson D, Hess D, Hill NS, Horan T, Kollef M, Levy M, Septimus E, VanAntwerpen C, Wright D, Lipsett P (2013) Developing a new, national approach to surveillance for ventilator-associated events*. Crit Care Med 41:2467-2475 